TITLE:

\title{
MicroRNA-133 regulates the expression of GLUT4 by targeting KLF15 and is involved in metabolic control in cardiac myocytes
}

\section{AUTHOR(S):}

Horie, Takahiro; Ono, Koh; Nishi, Hitoo; Iwanaga, Yoshitaka; Nagao, Kazuya; Kinoshita, Minako; Kuwabara, Yasuhide; ... Hasegawa, Koji; Kita, Toru; Kimura, Takeshi

\section{CITATION:}

Horie, Takahiro ... [et al]. MicroRNA-133 regulates the expression of GLUT4 by targeting KLF15 and is involved in metabolic control in cardiac myocytes. Biochemical and Biophysical Research Communications 2009, 389(2): 315-320

\section{ISSUE DATE:}

2009-11

URL:

http://hdl.handle.net/2433/86176

\section{RIGHT:}

c 2009 Elsevier Inc. All rights reserved.; この論文は出版社版でありませ ん。引用の際には出版社版をご確認ご利用ください。; This is not the published version. Please cite only the published version. 


\section{MicroRNA-133 regulates the expression of GLUT4 by targeting KLF15 and is involved in metabolic control in cardiac myocytes}

Takahiro Horie $^{1}$, Koh Ono ${ }^{1}$, Hitoo Nishi ${ }^{1}$, Yoshitaka Iwanaga ${ }^{2}$, Kazuya Nagao ${ }^{1}$, Minako Kinoshita ${ }^{1}$, Yasuhide Kuwabara ${ }^{1}$, Rieko Takanabe ${ }^{3}$, Koji Hasegawa ${ }^{3}$, Toru Kita $^{1}$ and Takeshi Kimura ${ }^{1}$

From:

Department of Cardiovascular Medicine, Graduate School of Medicine, Kyoto University, Kyoto, Japan ${ }^{1}$

Department of Cardiovascular Medicine, Graduate School of Medicine, Kinki University, Osakasayama, Japan²

Division of Translational Research, Kyoto Medical Center, National Hospital Organization, Kyoto, Japan ${ }^{3}$

Address for correspondence:

Koh Ono, MD, PhD,

Department of Cardiovascular Medicine, Kyoto University,

54 Shogoin-Kawaharacho, Sakyo-ku, Kyoto, 606-8507, Japan

Tel: +81-75-751-3190

Fax: +81-75-751-3203

E-mail: kohono@kuhp.kyoto-u.ac.jp

Running Title: Expression regulation of GLUT4 by miR-133 


\begin{abstract}
GLUT4 shows decreased levels in failing human adult hearts. We speculated that GLUT4 expression in cardiac muscle may be fine-tuned by microRNAs. Forced expression of miR-133 decreased GLUT4 expression and reduced insulin-mediated glucose uptake in cardiomyocytes. A computational miRNA target prediction algorithm showed that KLF15 is one of the targets of miR-133. It was confirmed that over-expression of miR-133 reduced the protein level of KLF15, which reduced the level of the downstream target GLUT4. Cardiac myocytes infected with lenti-decoy, in which the 3'UTR with tandem sequences complementary to miR-133 was linked to the luciferase reporter gene, had decreased miR-133 levels and increased levels of GLUT4. The expression levels of KLF15 and GLUT4 were decreased at the left ventricular hypertrophy and congestive heart failure stage in a rat model. The present results indicated that miR-133 regulates the expression of GLUT4 by targeting KLF15 and is involved in metabolic control in cardiomyocytes.
\end{abstract}




\section{Introduction}

Perturbed myocardial energetics is a potentially important and yet poorly studied mechanism for the progression of chronic heart failure (CHF), that has been reported in models of heart failure and cardiac hypertrophy [1]. The depletion of high energy phosphate metabolites may contribute to heart failure, and a decreased PCr/ATP ratio has been found in cardiac muscle of heart failure patients [2] and animal models of heart failure [3]. Myocardial glycolytic ATP production is important for preserving cardiac viability during ischemia [4]. A major determinant of glycolytic flux is glucose transport; glucose enters heart cells via the facilitative glucose transporters GLUT1 and GLUT4 [5]. GLUT4 resides in intracellular vesicles under basal conditions and translocates to the plasma membrane in response to insulin, ischemia, and exercise; hence, GLUT4 translocation represents the major mechanism by which glucose uptake into the cardiomyocytes can be increased [5]. Therefore, it is possible that maintenance of the level of GLUT4 is important for myocardial energy supply during cardiac hypertrophy and failure.

MicroRNAs (miRNAs) are small, non-protein-coding RNAs that recognize target sequences with imperfect complementarity in cognate mRNAs and inhibit protein translation. Growing evidence indicates that miRNAs affect pathways that are fundamental for metabolic control such as in adipocytes and skeletal muscle [6, 7].

Among $>500$ mammalian miRNAs identified thus far, miR-133 is believed to be expressed specifically in adult cardiac and skeletal muscle tissues where miR-133 regulates the differentiation and proliferation of these cells [8]. Recent studies suggested that miR-133 controls cardiac hypertrophy, and it is significantly down-regulated in hypertrophic and failing hearts [9-11]. Therefore, down-regulation of miR-133 may be 
involved in the development and progression of CHF. Because CHF patients have whole body insulin resistance [12-14], decreased myocardial fluorodeoxyglucose uptake during insulin clamp and decreased GLUT4 expression [15, 16], it was speculated that glucose uptake change in heart failure may be affected post-transcriptionally by miR-133. In the present study, miR-133 has reduced cardiomyocyte GLUT4 levels. KLF15 was established experimentally as a target for repression by miR-133. It was confirmed that overexpression of miR-133 reduced the protein level of KLF15 and reduced their downstream target GLUT4. Silencing endogenous miR-133 in vitro increased the levels of KLF15 and GLUT4, which indicated a potential role of miR-133 in cardiac myocytes.

The expression levels of GLUT4 and miR-133 were also investigated in Dahl salt-sensitive rats [17]. In this rat model under a high-salt diet, systemic hypertension caused compensated concentric left ventricular hypertrophy (LVH) at the age of 11 weeks, followed by marked LV dilatation and global hypokinesis at 17 weeks (CHF stage). The expression levels of KLF15 and GLUT4 in the heart were reduced at the LVH stage and further reduced at the CHF stage.

Thus, the present findings illustrated a novel function of miR-133 in fine-tuning KLF15-mediated cardiomyocyte GLUT4 expression. The down-regulation of miR-133a and 133b in failing hearts may contribute to the enhancing myocardial energy intake as a compensatory mechanism. 


\section{Materials and Methods}

\section{Reagents}

Anti-GLUT4 antibody (C-20) and anti-KLF15 antibody (N-16) were obtained from Santa Cruz Biotechnology (CA, USA). Anti- $\beta$ actin antibody (A5441) was obtained from Sigma Aldrich (Saint Louis, MI, USA). Anti-GAPDH antibody (14C10) was obtained from Cell Signaling Technology (Beverly, MA, USA).

\section{Isolation of neonatal rat cardiomyocytes}

The investigation conformed to the Guide for the Care and Use of Laboratory Animals published by the US National Institutes of Health (NIH Publication No. 85-23, revised 1996). Primary neonatal rat ventricular cardiomyocytes were prepared as described previously [18].

\section{Plasmids}

Expression vectors for the negative control and the microRNAs were generated using BLOCK-iT ${ }^{\mathrm{TM}}$ PolIImiR RNAi Expression Vector Kits following the manufacturer's protocol (Invitrogen). In order to create anti-miR-133 (decoy) vector, the luciferase 3’UTR was modified to include 3 tandem sequences complementary to miR-133a, separated by 3 nucleotides spacers [10]. Rat KLF15 was amplified from rat heart cDNA (BD Biosciences Clontech) using iProof DNA polymerase (Bio-Rad Laboratories, Inc.) and cloned into pcDNA3.1 (Invitrogen). The sequences of all constructs were analyzed using an ABI 3100 genetic analyzer. All of these constructs were correctly inserted into 
a pLenti6/V5-D-TOPO vector (Invitrogen) driven by a CMV promoter to stably express genes in neonatal rat cardiac myocytes.

\section{Lentivirus production and DNA transduction}

As described previously, lentiviral stocks were produced in 293FT cells following the manufacturer’s protocol (Invitrogen) [19, 20]. In brief, virus-containing medium was collected $48 \mathrm{~h}$ post transfection and filtered through a $0.45-\mu \mathrm{m}$ filter. One round of lentiviral infection was performed by replacing the medium with virus-containing medium (containing $8 \mu$ g of Polybrene ${ }^{\circledR}$ per ml), followed by centrifugation at 2,500 rpm for $30 \mathrm{~min}$ at $32^{\circ} \mathrm{C}$. Cells were used for analysis two or three days after DNA transduction.

\section{RNA extraction and quantitative real-time PCR for $\mathrm{mRNA}$}

Total RNA was isolated using TRIzol ${ }^{\circledR}$ reagent (Invitrogen) and cDNA was synthesized by using SuperScript II reverse transcriptase (Invitrogen). For real-time PCR, the reaction was performed with a SYBR Green PCR master mix (Applied Biosystems), and the products were analyzed using a thermal cycler (ABI Prism 7900HT sequence detection system). The Levels of GAPDH transcripts was used to normalize cDNA levels. Gene-specific primers were shown in supplementary methods.

\section{Western blotting}

Cell lysates were prepared as described previously and subjected to sodium dodecyl sulfate polyacrylamide gel electrophoresis, followed by standard western blotting procedures [19]. 


\section{Quantitative real-time PCR for microRNA}

For quantitative real-time PCR for microRNA, total RNA was reverse-transcribed into cDNA using a mirVana ${ }^{\mathrm{TM}}$ qRT-PCR miRNA Detection Kit (Ambion) and miRNA specific primers (mirVana ${ }^{\mathrm{TM}}$ qRT-PCR miRNA Primer Sets; Ambion). SYBR Green I Stain (Lonza, ME, USA) was used to monitor the amplification of cDNA, and ROX Reference Dye (Invitrogen) was used for normalization. Super Taq ${ }^{\mathrm{TM}}$ (Ambion) was employed to amplify cDNA. Amplification was performed using an ABI Prism 7900HT sequence detection system (Applied Biosystems). Samples were normalized by $5 \mathrm{~S}$ rRNA expression levels.

\section{Northern blotting for microRNA}

Small RNA fractions were isolated from total RNA using a mirVana miRNA Isolation kit (Ambion). The small RNA fractions $5 \mu$ g were separated by electrophoresis using 15\% polyacrylamide (19:1) denaturing gel and transferred to nylon hybridization membrane (Hybond-NX ; Amersham) using a semidry electroblotter (Bio-Rad, Hercules, CA, USA) at $20 \mathrm{~V}$ for $30 \mathrm{~min}$ at $4^{\circ} \mathrm{C}$. Cross-linking of RNA was performed using 0.16 M 1-ethyl-3-(3-dimethylaminopropyl) carbodiimide (EDC; Sigma) in 0.13 M 1-methylimidazole (Sigma) at $\mathrm{pH} 8.0$ for $2 \mathrm{hr}$ at $60^{\circ} \mathrm{C}$. Templates to make probes for miR-133a, miR-133b, and U6 were prepared using a mirVana miRNA Probe Construction Kit (Ambion) with the following oligonucleotides:

MiR-133a; TTGGTCCCCTTCAACCAGCTGTCCTGTCTC MiR-133b; TTGGTCCCCTTCAACCAGCTACCTGTCTC U6; CGATACAGAGAAGATTAGCATGGCCCCTGCCCTGTCTC 
Transcription of DNA templates was carried out with 20-40 U of T7 RNA polymerase per reaction and $\alpha-{ }_{-}^{32} \mathrm{P}$ UTP (800Ci/mmol; Amarsham). Hybridizations were performed in ULTRAhyb solution (Ambion). All radioisotopic images were recorded using a BAS-5000 system (Fuji Film) with Multi Gauge Ver3.0 Image Analyzer software.

\section{Dual-luciferase assays and lipofection}

For GLUT4 promoter assays, a pGL3-basic (Promega, Madison, WI, USA) reporter plasmid was used containing the rat GLUT4 gene promoter (1000 bp nucleotide). Mutations in the KLF15 binding site were introduced using a QuikChange ${ }^{\mathrm{TM}}$ Site-Directed Mutagenesis Kit (Invitrogen) in accordance with the instruction manual. Cells were plated in 24-well plates and transiently transfected with firefly luciferase reporter plasmid $(0.1 \mu \mathrm{g})$ and expression vectors $(0.5 \mu \mathrm{g})$ using Lipofectamine ${ }^{\mathrm{TM}}$ Reagent (Invitrogen). A fixed amount $(0.01 \mu \mathrm{g})$ of internal control reporter, Renilla reniformis luciferase, driven by the thymidine kinase (TK) promoter (pRL-TK: Promega) was also co-transfected to normalize the transfection efficiency. At $48 \mathrm{~h}$ after transfection, luciferase activities were measured using a dual luciferase kit (PicaGene ${ }^{\circledR}$ dual kit, Toyo Ink Co.). The relative luciferase activity of each construct (arbitrary unit) was reported as the fold induction

\section{Glucose uptake assays}

Cardiomyocytes were grown in 24-well plates. Three days after DNA transfection using lenti virus vector, 2-deoxyglucose (2DG) uptake was determined, as described previously [19]. 2-Deoxy-D-[1- $\left.{ }^{3} \mathrm{H}\right]$ glucose was obtained from Amersham Biosciences. 


\section{Statistics}

Data are presented as means \pm S.E. Statistical comparisons were performed using unpaired two-tailed Student's t-tests or one-way analysis of variance with Bonferonni’s post hoc test where appropriate, with a probability value of $<0.05$ was taken to indicate significance.

Note: Supplementary methods are available on website 


\section{Results}

\section{MicroRNA-133 reduced the expression of GLUT4}

First, an attempt was made to find a link between miR-133 and glucose transporters. Expression vectors for negative control and microRNAs were generated using BLOCK-iT ${ }^{\mathrm{TM}}$ PolIImiR RNAi Expression Vector Kits following the manufacturer's protocol (Invitrogen). Fig.1A shows the expression level of miR-133a by Northern blotting in neonatal rat cardiac myocytes. The transduction efficiency of these miRNAs was always over 90\% (Supplement Fig.1A). The over-expression of miR-133a and 133b decreased cardiomyocyte cell surface area as described previously (Supplement Fig.1A and 1B) [10]. Fig.1B and 1C indicate that over-expression of miR-133a and 133b reduced the mRNA and protein levels of GLUT4, but they had no effect on GLUT1 expression (Supplement Fig.1C). The effect of these miR expressions on 2-deoxyglucose (2DG) uptake was also measured in cardiac myocytes. Although the expression of miR-133a or 133b did not change the basal levels of 2-DG uptake, they reduced insulin-induced glucose uptake significantly (Fig.1D).

\section{KLF15 is a target of miR-133 in cardiac myocytes}

The computational miRNA target prediction algorithm showed that KLF15 is a target of miR-133 (MiRNAMap http://mirnamap.mbc.nctu.edu.tw/, Supplement Fig.2A). The expression of 133a and 133b reduced the protein levels of KLF15, whereas they did not change the KLF15 mRNA level (Fig.2A and Supplement Fig.2B). The rat GLUT4 promoter was isolated by PCR and cloned upstream of a luciferase reporter gene. Point-mutations of the potential KLF15 binding site reduced the expression of luciferase in cardiac myocytes (Fig.2B). This finding shows the endogenous contribution of 
KLF15 to GLUT4 expression. Moreover, the expression of miR-133a and 133b reduced the expression levels of the luciferase reporter gene (Fig.2C).

\section{Reduction in endogenous miR-133 using forced expression of a 'decoy' gene}

To assess the functional consequences of silencing endogenous miR-133 and the miRNAs with the same seed sequence in vitro, cardiac myocytes infected with a lentivirus vector were used, in which the 3'UTR with three tandem sequences complementary to miR-133 was linked to the luciferase reporter gene (miR-133 decoy, Supplement Fig.3A). The complementary sequences acted as a decoy, sequestering endogenous miR-133 and other miRNAs that have the same seed sequence. When the same amount of control and miR-133 decoy were transduced into cardiomyocytes, the luciferase activity was significantly reduced (Supplement Fig.3B). Northern blot analysis indicated that the miR-133a level was significantly reduced by the expression of miR-133 decoy (Fig.3A). The expression of miR-133 decoy enhanced the protein level of KLF15 without changing its mRNA level (Fig.3B, Supplement Fig.3C and Supplement Fig.3D). The expression of miR-133 decoy further enhanced both the protein and mRNA level of GLUT4 in cardiac myocytes (Fig. 3C and Supplement Fig.3E).

\section{Analysis of Dahl salt-sensitive rat hearts}

Hemodynamic overload in the heart can trigger maladaptive hypertrophy of cardiac myocytes. In salt-sensitive Dahl rats on a high-salt diet, systemic hypertension caused compensated concentric left ventricular hypertrophy (LVH) at the age of 11 weeks (LVH stage), followed by marked LV dilatation and global hypokinesis at 17 weeks (CHF stage). The expression levels of ANF were enhanced in the LVH stage and 
increased dramatically in the CHF stage (Fig. 4A). The expression levels of KLF15 and GLUT4 were investigated in this model. These expression levels decreased at the LVH stage and further decreased at the CHF stage (Figs. 4B and C). KLF15 protein level is indicated in Fig. 4D. The expression levels of miR-133a and 133b were parallel to these levels (Figs 4E and F). 


\section{Discussion}

Cardiac diseases are a major cause of morbidity and mortality worldwide. Hypertension and myocardial infarction, in particular, predispose patients the development of heart failure. Changes in cardiac substrate utilization and energy metabolism, including a decline in high-energy-phosphate content, a reduction in fatty acid oxidation rate, and an increased dependence on glucose as a substrate, are hallmarks of a hypertrophied and failing heart [21, 22]. Although the question as to whether altered substrate metabolism is a cause or consequence of cardiac failure is still unsolved, the decline in cardiac function could be caused in part by diminished substrate oxidation.

Because of its hydrophilic nature, glucose is unable to pass the lipid bilayer of the plasma membrane by simple diffusion. In cardiomyocytes two members of the glucose transporter GLUT family are present, GLUT1 and GLUT4 [23, 24]. While GLUT1 is regarded as the basal glucose transporter, GLUT4 is responsible for the increase in glucose uptake upon insulin stimulation and oxidative stress [19].

MicroRNA-mediated gene regulation is now considered a fundamental layer of genetic programs that operate at the posttranscriptional level. However, despite an ability to identify miRNAs, regulatory targets have not been established for any of the vertebrate miRNAs. Current understanding of the function of these miRNAs is mainly derived from the computational target prediction and information about its developmental expression pattern as well as their evolutionary conservation.

Recently, the developmental role of miR-133 was shown in miR-133-null mice [25]. The study revealed that miR-133 is essential in orchestrating cardiac development and the SRF-dependent myogenic transcriptional circuit. However, its potential role in 
heart failure still remains unknown. Because it is already reported that miR-133 is repressed in diseased hearts [9-11], it was speculated that the suppression of miR-133 may have a role in maintaining the level of GLUT4 in cardiac myocytes. This report identified that one of the targets of miR-133 is KLF15, which is important for the transcription of GLUT4.

A major form of GLUT4 regulation involves its translocation from the interior of cells to the plasma membrane [26]. However, GLUT4 mRNA levels are also regulated in conditions such as experimental diabetes and fasting [27]. In addition, it was demonstrated that perinatal expression of cardiac GLUT4 is controlled directly at the level of gene transcription [28]. Thus, an understanding of the mechanisms governing GLUT4 gene expression has been of considerable interest. Previous studies identified a proximal regulatory region that contains a critical MEF-binding element [29, 30]. We also observed that MEF2C is downregulated by miR-133 overexpression. However, miR-133 decoy did not affect MEF2C level (data not shown). Gray et al. found that KLF15 strongly induces the GLUT4 promoter and that the majority of this activity was mediated by a binding site that lies in proximity to the MEF site [31]. Our results also provide evidences that KLF15 is important for GLUT4 expression and that there is another complex regulation of GLUT4 by posttranslational regulation of KLF15 via miR-133 in the heart.

Recently, KLF15 deficient mice were generated by Fish et al. [32] and they indicated that there weren't any significant difference in GLUT4mRNA between KLF15 $(+/+)$ vs. (-/-) hearts. However, miR-133 is expressed only in myocytes in the heart, in vivo regulation of GLUT4 by miR-133 occurs selectively in cardiac myocytes, which is completely different to the situation of KLF (-/-) hearts. It is also possible that there may 
be some other compensatory mechanisms for the GLUT4 expression in the whole heart when KLF15 is ablated by gene targeting.

The present findings illustrate a novel function of miR-133 in fine-tuning KLF15-mediated GLUT4 expression. The function of miR-133 in the heart may be complex and further experiment is required to the understanding of its overall function in cardiac diseases. 


\section{Acknowledgements}

We thank Naoya Sowa and Akemi Fukumoto for excellent technical help.

\section{Sources of Funding}

This work was supported in part by a Grant-in-Aid for Scientific Research from the Ministry of Education, Culture, Sports, Science and Technology of Japan to K. Ono, K. Hasegawa and T. Kita. 


\section{Figure legend}

\section{Figure 1}

\section{MicroRNA-133 reduced the expression of GLUT4.}

A. Northern blotting analysis for miR-133a in miR-control and miR-133a over-expressed cardiac myocytes.

B. Quantitative real-time PCR analysis for GLUT4 in miR-133 over-expressing cardiac myocytes. Values are the means \pm S.E. of 5-6 independent experiments $\left({ }^{*} \mathrm{p}<0.05\right.$ vs. miR-control).

C. Representative western blotting analysis for the expression of GLUT4 in miR-133 over-expressed cardiac myocytes.

D. 2-DG uptake in basal and insulin-stimulated states (10 min). The mean level of 2-DG uptake without treatment in miR-control group was set at $100 \%$. Values are the means \pm S.E. of 4 independent experiments. ( ${ }^{\star \star} \mathrm{P}<0.01$ vs. miR-control)

\section{Figure 2}

\section{KLF15 is one of targets of miR-133 in cardiac myocytes.}

A. Representative western blotting analysis for the expression of KLF15.

B. GLUT4 promoter analysis was performed in cardiac myocytes. The same amounts of reporter genes (wild-type or mutant) were introduced and luciferase activity was measured as mentioned in material and methods. Mutations in the KLF15 binding sites reduced GLUT4 promoter activity. Values are the means \pm S.E. of 3 independent experiments. ( $\left.{ }^{\star \star} \mathrm{P}<0.01\right)$

C. GLUT4 promoter luciferase activity was measured 2 days after co-transfection. Values are the means \pm S.E. of 4 independent experiments $\left({ }^{*} \mathrm{p}<0.05\right.$ vs. miR-control and empty vector).

\section{Figure 3}

\section{Reduction of endogenous miR-133 using forced expression of a 'decoy' gene.}

A. Northern blotting analysis for miR-133a. Lentivirus-mediated forced expression of the 'decoy' gene reduced endogenous microRNA-133a levels.

B. Representative western blotting analysis for KLF15 in cardiac myocytes after lentivirus infection with control or 'decoy' genes. GAPDH was used as a loading control.

C. Representative western blotting analysis for GLUT4 of cardiac myocytes after lentivirus infection with the control or 'decoy' gene. GAPDH was used as a loading 
control.

\section{Figure 4}

Analysis of Dahl salt-sensitive rat heart

A. B. C. Dahl salt-sensitive rats suffer from systemic hypertension with a high salt diet. Systemic hypertension causes compensated concentric left ventricular hypertrophy $(\mathrm{LVH})$ at the age of 11 weeks, followed by marked LV dilatation and global hypokinesis (CHF) at the age of 17 weeks (11WLS; 11 weeks low salt, 17WLS; 17 weeks low salt). The expression levels of ANF (A), GLUT4 (B), and KLF15 (C) in each stage were measured using quantitative real-time PCR analysis. The mean values at 11WLS, normalized with GAPDH, were set at 1.0. Values are the means \pm S.E. of 5-6 independent experiments $\left({ }^{*} \mathrm{p}<0.05\right.$ vs. $11 \mathrm{WLS},{ }^{* *} \mathrm{p}<0.01$ vs. 11WLS, \#\#p<0.01 vs. 17WLS, $++p<0.01$ vs. LVH).

D. Representative western blotting results for KLF15 in Dahl salt-sensitive rat heart.

E. F. Quantitative analysis for microRNA-133a (E) and microRNA-133b

(F) by real-time PCR. The mean values of $11 \mathrm{WLS}$, normalized with $5 \mathrm{~S}$ rRNA were set at 1.0. Values are the means \pm S.E. of 5 independent experiments $\left({ }^{*} \mathrm{p}<0.05\right.$ vs. $11 \mathrm{WLS},{ }^{\star \star} \mathrm{p}<0.01$ vs. $11 \mathrm{WLS}, \# \# \mathrm{p}<0.01$ vs. $17 \mathrm{WLS},+\mathrm{p}<0.05$ vs. $\left.L V H\right)$ 


\section{Reference}

[1] Marin-Garcia, J., Goldenthal, M. J., and Moe, G. W., Mitochondrial pathology in cardiac failure, Cardiovasc Res 49 (2001) 17-26.

[2] Neubauer, S., Krahe, T., Schindler, R., Horn, M., Hillenbrand, H., Entzeroth, C., Mader, H., Kromer, E. P., Riegger, G. A., Lackner, K., and et al., 31P magnetic resonance spectroscopy in dilated cardiomyopathy and coronary artery disease. Altered cardiac high-energy phosphate metabolism in heart failure, Circulation 86 (1992) 1810-1818.

[3] Horn, M., Remkes, H., Stromer, H., Dienesch, C., and Neubauer, S., Chronic phosphocreatine depletion by the creatine analogue beta-guanidinopropionate is associated with increased mortality and loss of ATP in rats after myocardial infarction, Circulation 104 (2001) 1844-1849.

[4] Cross, H. R., Opie, L. H., Radda, G. K., and Clarke, K., Is a high glycogen content beneficial or detrimental to the ischemic rat heart? A controversy resolved, Circ Res 78 (1996) 482-491.

[5] Tian, R., and Abel, E. D., Responses of GLUT4-deficient hearts to ischemia underscore the importance of glycolysis, Circulation 103 (2001) 2961-2966.

[6] Takanabe, R., Ono, K., Abe, Y., Takaya, T., Horie, T., Wada, H., Kita, T., Satoh, N., Shimatsu, A., and Hasegawa, K., Up-regulated expression of microRNA-143 in association with obesity in adipose tissue of mice fed high-fat diet, Biochem Biophys Res Commun 376 (2008) 728-732.

[7] Krutzfeldt, J., and Stoffel, M., MicroRNAs: a new class of regulatory genes affecting metabolism, Cell Metab 4 (2006) 9-12.

[8] Chen, J. F., Mandel, E. M., Thomson, J. M., Wu, Q., Callis, T. E., Hammond, S. M., Conlon, F. L., and Wang, D. Z., The role of microRNA-1 and microRNA-133 in skeletal muscle proliferation and differentiation, Nat Genet 38 (2006) 228-233.

[9] van Rooij, E., Sutherland, L. B., Liu, N., Williams, A. H., McAnally, J., Gerard, R. D., Richardson, J. A., and Olson, E. N., A signature pattern of stress-responsive microRNAs that can evoke cardiac hypertrophy and heart failure, Proc Natl Acad Sci U S A 103 (2006) 18255-18260.

[10] Care, A., Catalucci, D., Felicetti, F., Bonci, D., Addario, A., Gallo, P., Bang, M. L., Segnalini, P., Gu, Y., Dalton, N. D., Elia, L., Latronico, M. V., Hoydal, M., 
Autore, C., Russo, M. A., Dorn, G. W., 2nd, Ellingsen, O., Ruiz-Lozano, P., Peterson, K. L., Croce, C. M., Peschle, C., and Condorelli, G., MicroRNA-133 controls cardiac hypertrophy, Nat Med 13 (2007) 613-618.

[11] Sayed, D., Hong, C., Chen, I. Y., Lypowy, J., and Abdellatif, M., MicroRNAs play an essential role in the development of cardiac hypertrophy, Circ Res $\mathbf{1 0 0}$ (2007) 416-424.

[12] Paolisso, G., De Riu, S., Marrazzo, G., Verza, M., Varricchio, M., and D'Onofrio, F., Insulin resistance and hyperinsulinemia in patients with chronic congestive heart failure, Metabolism 40 (1991) 972-977.

[13] Swan, J. W., Anker, S. D., Walton, C., Godsland, I. F., Clark, A. L., Leyva, F., Stevenson, J. C., and Coats, A. J., Insulin resistance in chronic heart failure: relation to severity and etiology of heart failure, J Am Coll Cardiol 30 (1997) 527-532.

[14] Kemppainen, J., Tsuchida, H., Stolen, K., Karlsson, H., Bjornholm, M., Heinonen, O. J., Nuutila, P., Krook, A., Knuuti, J., and Zierath, J. R., Insulin signalling and resistance in patients with chronic heart failure, $J$ Physiol 550 (2003) 305-315.

[15] Paternostro, G., Camici, P. G., Lammerstma, A. A., Marinho, N., Baliga, R. R., Kooner, J. S., Radda, G. K., and Ferrannini, E., Cardiac and skeletal muscle insulin resistance in patients with coronary heart disease. A study with positron emission tomography, J Clin Invest 98 (1996) 2094-2099.

[16] Paternostro, G., Pagano, D., Gnecchi-Ruscone, T., Bonser, R. S., and Camici, P. G., Insulin resistance in patients with cardiac hypertrophy, Cardiovasc Res $\mathbf{4 2}$ (1999) 246-253.

[17] Inoko, M., Kihara, Y., Morii, I., Fujiwara, H., and Sasayama, S., Transition from compensatory hypertrophy to dilated, failing left ventricles in Dahl salt-sensitive rats, Am J Physiol 267 (1994) H2471-2482.

[18] Inada, T., Fujiwara, H., Hasegawa, K., Araki, M., Yamauchi-Kohno, R., Yabana, H., Fujiwara, T., Tanaka, M., and Sasayama, S., Upregulated expression of cardiac endothelin-1 participates in myocardial cell growth in Bio14.6 Syrian cardiomyopathic hamsters, J Am Coll Cardiol 33 (1999) 565-571.

[19] Horie, T., Ono, K., Nagao, K., Nishi, H., Kinoshita, M., Kawamura, T., Wada, H., Shimatsu, A., Kita, T., and Hasegawa, K., Oxidative stress induces GLUT4 translocation by activation of PI3-K/Akt and dual AMPK kinase in cardiac myocytes, J Cell Physiol 215 (2008) 733-742.

[20] Horie, T., Ono, K., Kinoshita, M., Nishi, H., Nagao, K., Kawamura, T., Abe, Y., 
Wada, H., Shimatsu, A., Kita, T., and Hasegawa, K., TG-interacting factor is required for the differentiation of preadipocytes, J Lipid Res 49 (2008) 1224-1234.

[21] Ventura-Clapier, R., Garnier, A., and Veksler, V., Energy metabolism in heart failure, J Physiol 555 (2004) 1-13.

[22] Stanley, W. C., Recchia, F. A., and Lopaschuk, G. D., Myocardial substrate metabolism in the normal and failing heart, Physiol Rev 85 (2005) 1093-1129.

[23] Becker, C., Sevilla, L., Tomas, E., Palacin, M., Zorzano, A., and Fischer, Y., The endosomal compartment is an insulin-sensitive recruitment site for GLUT4 and GLUT1 glucose transporters in cardiac myocytes, Endocrinology 142 (2001) 5267-5276.

[24] Fischer, Y., Thomas, J., Sevilla, L., Munoz, P., Becker, C., Holman, G., Kozka, I. J., Palacin, M., Testar, X., Kammermeier, H., and Zorzano, A., Insulin-induced recruitment of glucose transporter 4 (GLUT4) and GLUT1 in isolated rat cardiac myocytes. Evidence of the existence of different intracellular GLUT4 vesicle populations, J Biol Chem 272 (1997) 7085-7092.

[25] Liu, N., Bezprozvannaya, S., Williams, A. H., Qi, X., Richardson, J. A., Bassel-Duby, R., and Olson, E. N., microRNA-133a regulates cardiomyocyte proliferation and suppresses smooth muscle gene expression in the heart, Genes Dev 22 (2008) 3242-3254.

[26] Shepherd, P. R., and Kahn, B. B., Glucose transporters and insulin action--implications for insulin resistance and diabetes mellitus, $N$ Engl $J$ Med 341 (1999) 248-257.

[27] Katz, E. B., Burcelin, R., Tsao, T. S., Stenbit, A. E., and Charron, M. J., The metabolic consequences of altered glucose transporter expression in transgenic mice, J Mol Med 74 (1996) 639-652.

[28] Gerrits, P. M., Olson, A. L., and Pessin, J. E., Regulation of the GLUT4/muscle-fat glucose transporter mRNA in adipose tissue of insulin-deficient diabetic rats, J Biol Chem 268 (1993) 640-644.

[29] Thai, M. V., Guruswamy, S., Cao, K. T., Pessin, J. E., and Olson, A. L., Myocyte enhancer factor 2 (MEF2)-binding site is required for GLUT4 gene expression in transgenic mice. Regulation of MEF2 DNA binding activity in insulin-deficient diabetes, J Biol Chem 273 (1998) 14285-14292.

[30] Charron, M. J., Katz, E. B., and Olson, A. L., GLUT4 gene regulation and manipulation, J Biol Chem 274 (1999) 3253-3256.

[31] Gray, S., Feinberg, M. W., Hull, S., Kuo, C. T., Watanabe, M., Sen-Banerjee, S., 
DePina, A., Haspel, R., and Jain, M. K., The Kruppel-like factor KLF15 regulates the insulin-sensitive glucose transporter GLUT4, J Biol Chem 277 (2002) 34322-34328.

[32] Fisch, S., Gray, S., Heymans, S., Haldar, S. M., Wang, B., Pfister, O., Cui, L., Kumar, A., Lin, Z., Sen-Banerjee, S., Das, H., Petersen, C. A., Mende, U., Burleigh, B. A., Zhu, Y., Pinto, Y. M., Liao, R., and Jain, M. K., Kruppel-like factor 15 is a regulator of cardiomyocyte hypertrophy, Proc Natl Acad Sci U S A 104 (2007) 7074-7079. 


\section{Supplemental methods}

\section{Immunocytochemistry and measurement of cell size}

Cardiac myocytes were grown in flask-style chambers with glass slides (Nalgen Nunc, Naperville, IL, USA). Two days after lenti virus infection, the cells were fixed with $10 \%$ formaldehyde in phosphate-buffered saline (PBS) for $10 \mathrm{~min}$ at room temperature. Immunocytochemical staining for $\beta$-myosin heavy chain ( $\beta$-MHC) was performed using an indirect immunoperoxidase method. The anti-cardiac $\beta$-MHC polyclonal antibody (NovoCastra, Newcastle, UK) was used at a dilution of 1:50. A total of 50 cells were selected at random from cardiac myocytes stained with anti- $\beta$-MHC antibody, and the surface areas of these cells were measured with the aid of an image analyzer (Image-J software).

\section{Primer sequences for real-time PCR}

GLUT1 sense; 5’AATGCGGACTTGTGGCCTCT3’

GLUT1 antisense; 5'GCAGAAGGGCAACAGGATAC3'

GLUT4 sense; 5’TGGCATGGGTTTCCAGTATG3’

GLUT4 antisense; 5’GGTTTCACCTCCTGCTCTAA3’

KLF15 sense; 5’GCCTTCTGTTCCTGCTAC3’

KLF15 antisense; 5’TCCTGCTTCACAGCAACAG3’

ANF sense; 5’TTCCTCGTCTTGGCCTTTTG3’

ANF antisense; 5’CCTCATCTTCTACCGGCATCTTC3’

GAPDH sense; 5’TTGCCATCAACGACCCCTTC3’

GAPDH antisense; 5’TTGTCATGGATGACCTTGGC3’ 


\section{Supplemental Figure legends}

\section{Supplemental Figure 1}

A. MiR-control (negative control) and miR-133 were transduced into neonatal rat cardiomyocytes using lentivirus vector. The transduction efficiency, which is shown by GFP in the middle pannel, was always over $90 \%$. Immunocytochemical staining of $\beta$-MHC is shown in the lower panels

B. Measurement of cell surface area of miR-control (negative control) and miR-133 over-expressing cardiac myocytes. The cell surface area was measured for 50 randomly selected cardiac myocytes in each group. Values are expressed in terms of arbitrary units $\left({ }^{*} \mathrm{p}<0.05\right.$ vs. miR-control).

C. Quantitative real-time PCR analysis showed no significant change in mRNA level of GLUT1 in miR-133 over-expressing cardiac myocytes. Values are the means \pm S.E. of 5-6 independent experiments (n.s.; not significant).

\section{Supplemental Figure 2}

A. MiR-133 target sites are preserved in human, rat, and mouse 3'UTR of KLF15 (Mmu: mouse, Rno: rat, and Hsa: human).There are 2 potential binding site in 3’UTR of KLF15.

B. Quantitative real-time PCR analysis showed no significant change in mRNA level of KLF15 in miR-133 over-expressing cardiac myocytes. Values are the means \pm S.E. of 4 independent experiments (n.s.; not significant).

\section{Supplemental Figure 3}

A. Scheme of the 'decoy' gene. The luciferase 3'UTR was modified to include 3 tandem sequences complementary to miR-133a separated by 3 nucleotides spacers.

B. The same amount $(0.5 \mu \mathrm{g})$ of plasmid (a; control / b; decoy) was transfected into cardiac myocytes and luciferase activity was measured. This reduction was considered as the effect of the 'decoy' gene. The mean level of luciferase activity in the control group was set at $100 \%$. Values are the means \pm S.E. of 4 independent experiments $\left({ }^{* *} \mathrm{p}<0.01\right)$.

C. The densitometric analysis of KLF15 in cardiac myocytes after lentivirus infection with control or 'decoy' genes. Values are the means \pm S.E. of 4 independent experiments ( ${ }^{*} \mathrm{p}<0.05$ vs. control-decoy).

D. Quantitative real-time PCR analysis showed no significant changes in the mRNA level of KLF15 between cardiac myocytes after lentivirus infection with control or 
'decoy-miR-133’ gene (n.s.; not significant). Values are the means \pm S.E. of 8 independent experiments.

E. Quantitative real-time PCR analysis for GLUT4 in cardiac myocytes after lenti virus infection with control or 'decoy' genes. Values are the means \pm S.E. of 6 independent experiments ( ${ }^{*} \mathrm{p}<0.05$ vs. control-decoy). 


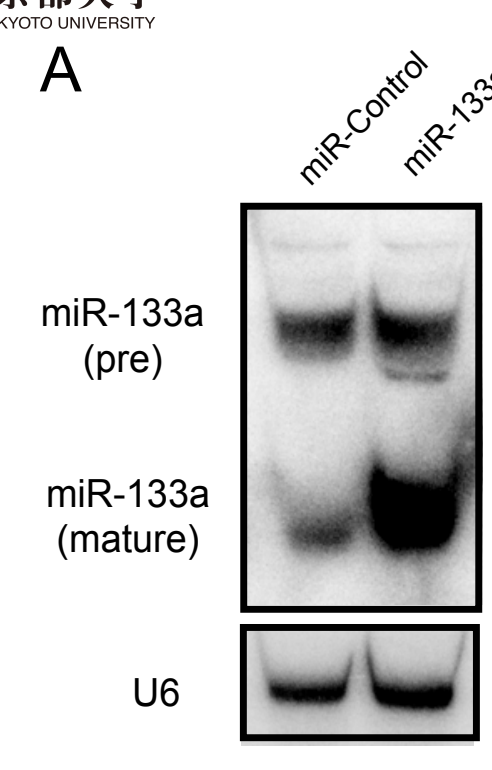

B

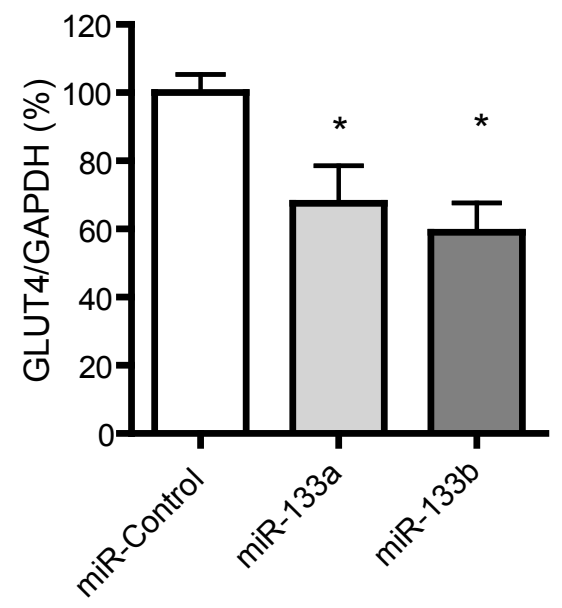

C

D

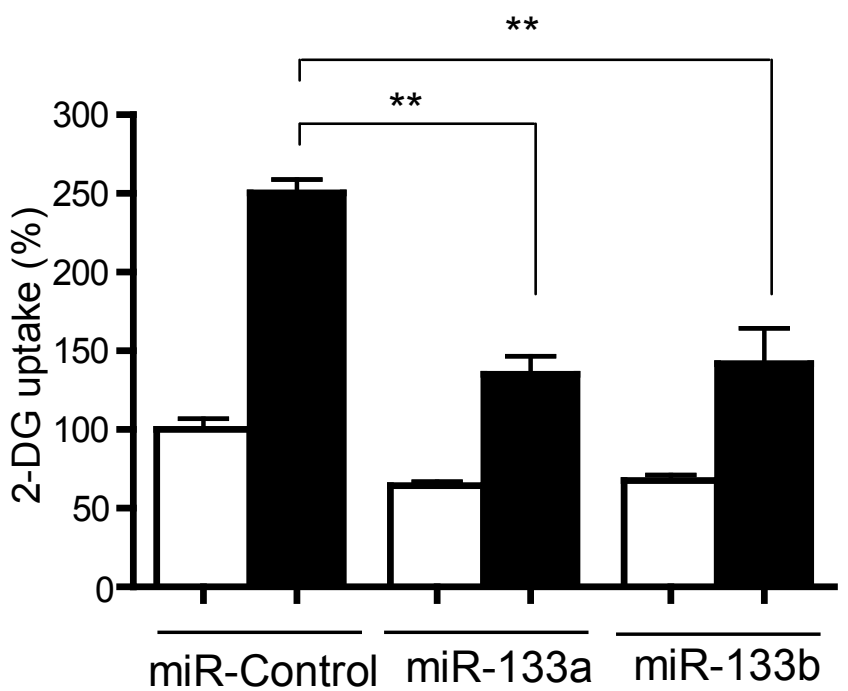

Fig.1 
A

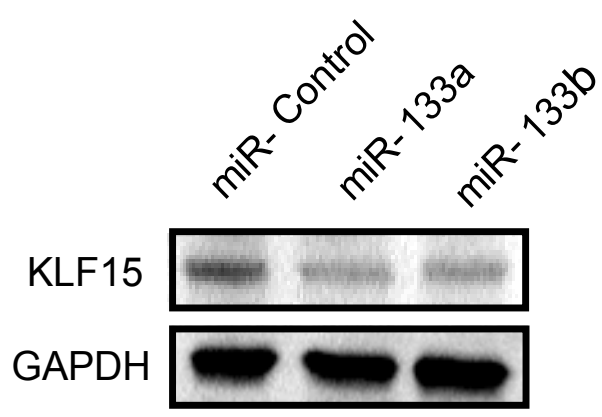

C

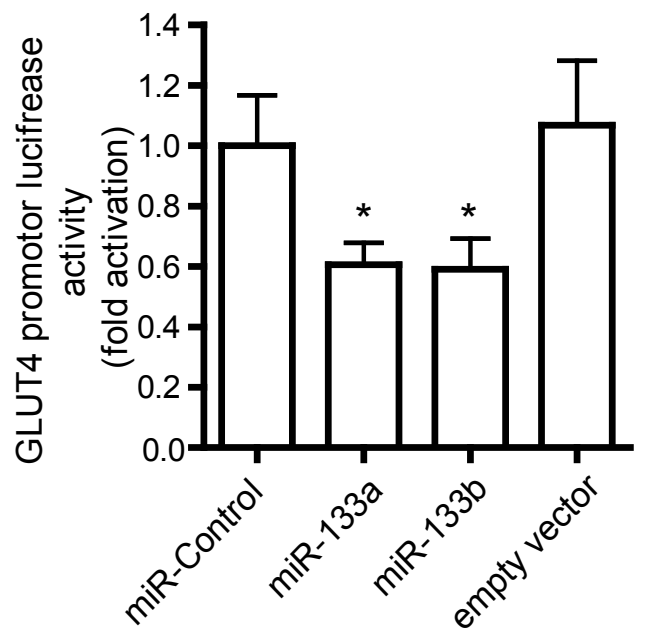

B

GLUT4 promotor(1000bp)
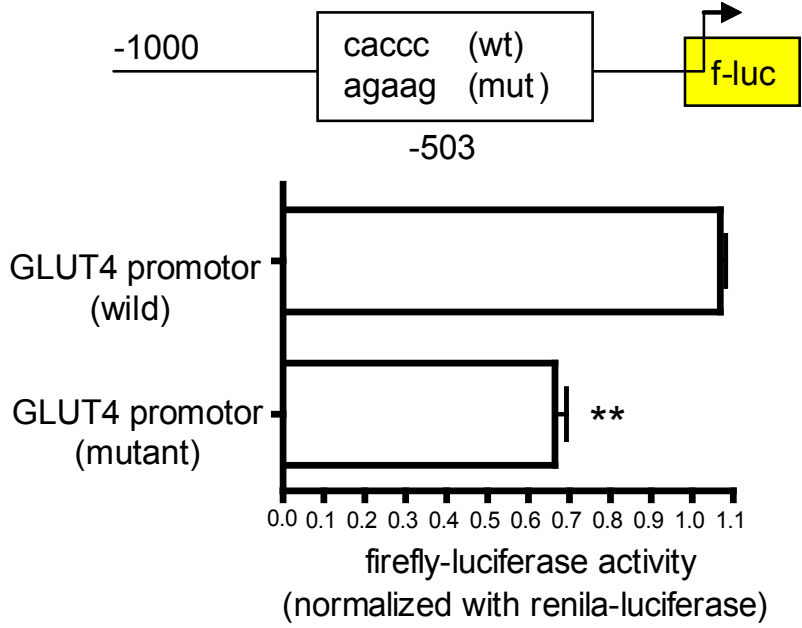

Fig.2 
A

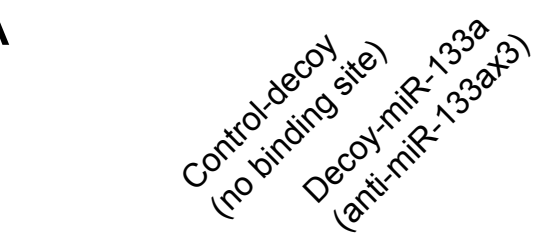

B
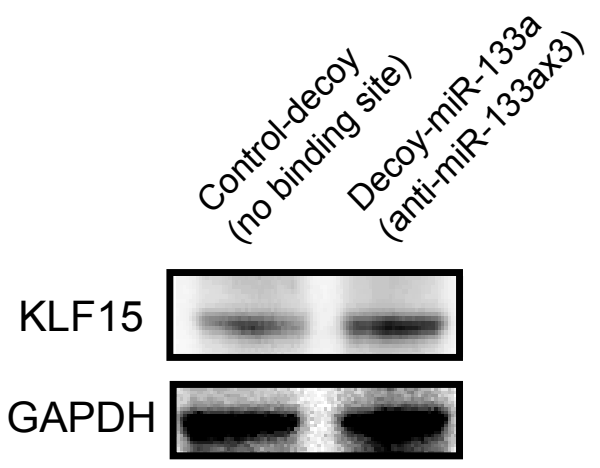

C

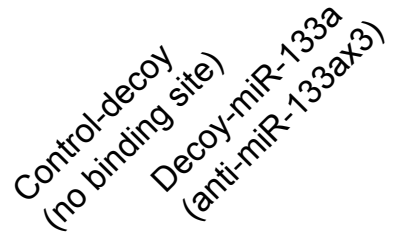

GLUT4

GAPDH
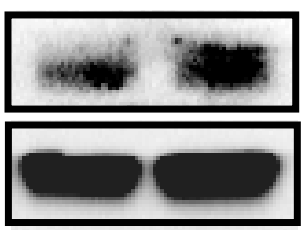

Fig.3 
A

B

C
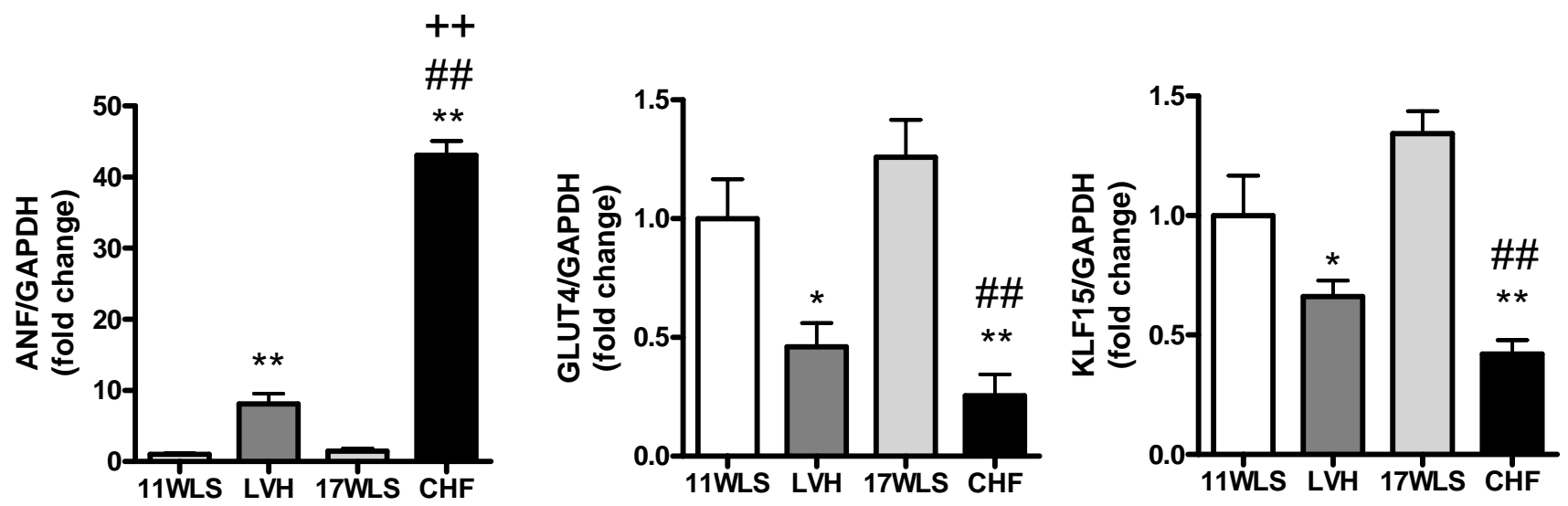

D

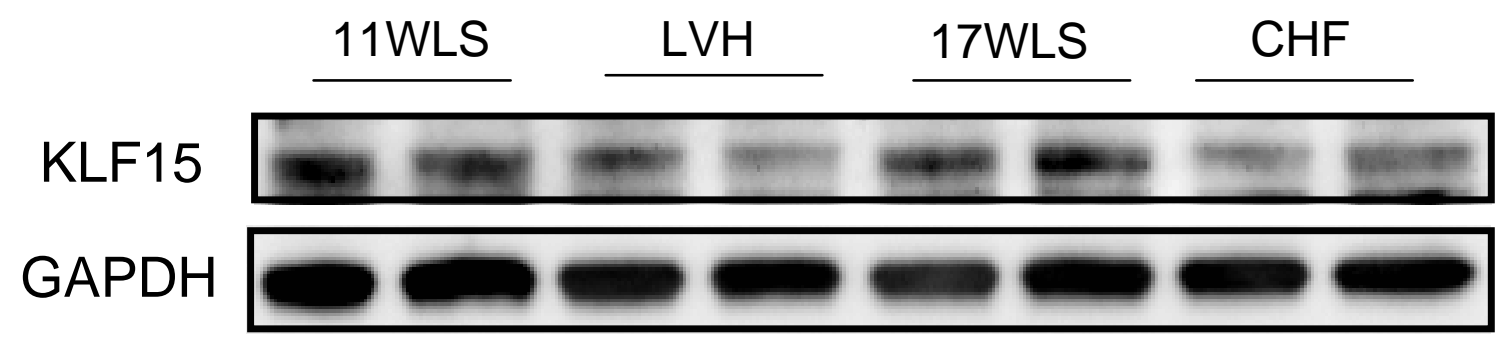

E

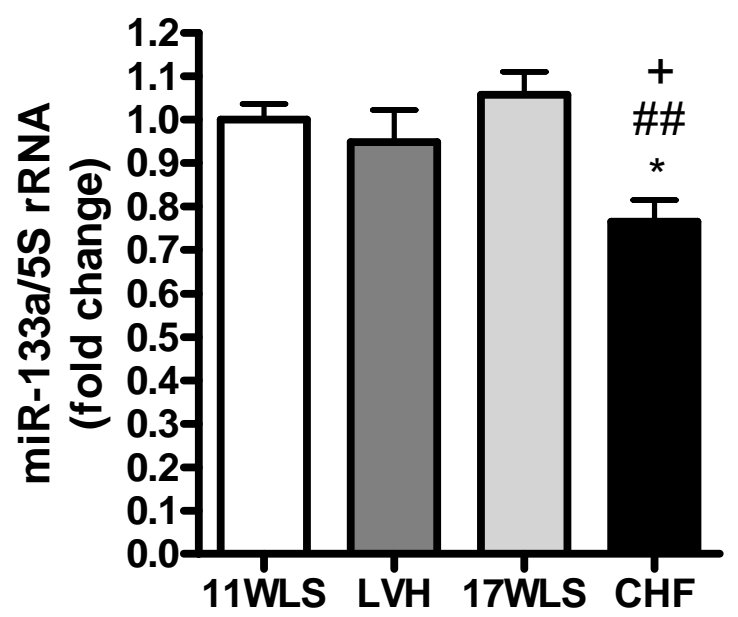

F

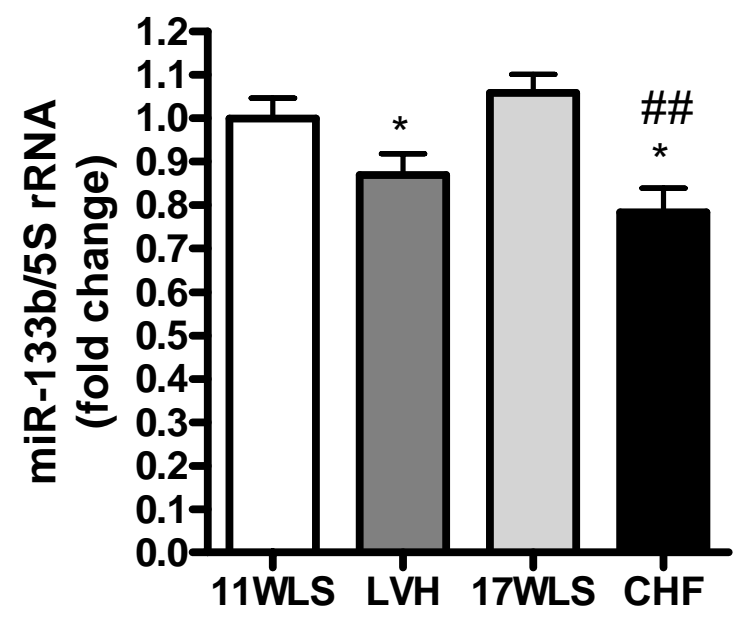

Fig.4 


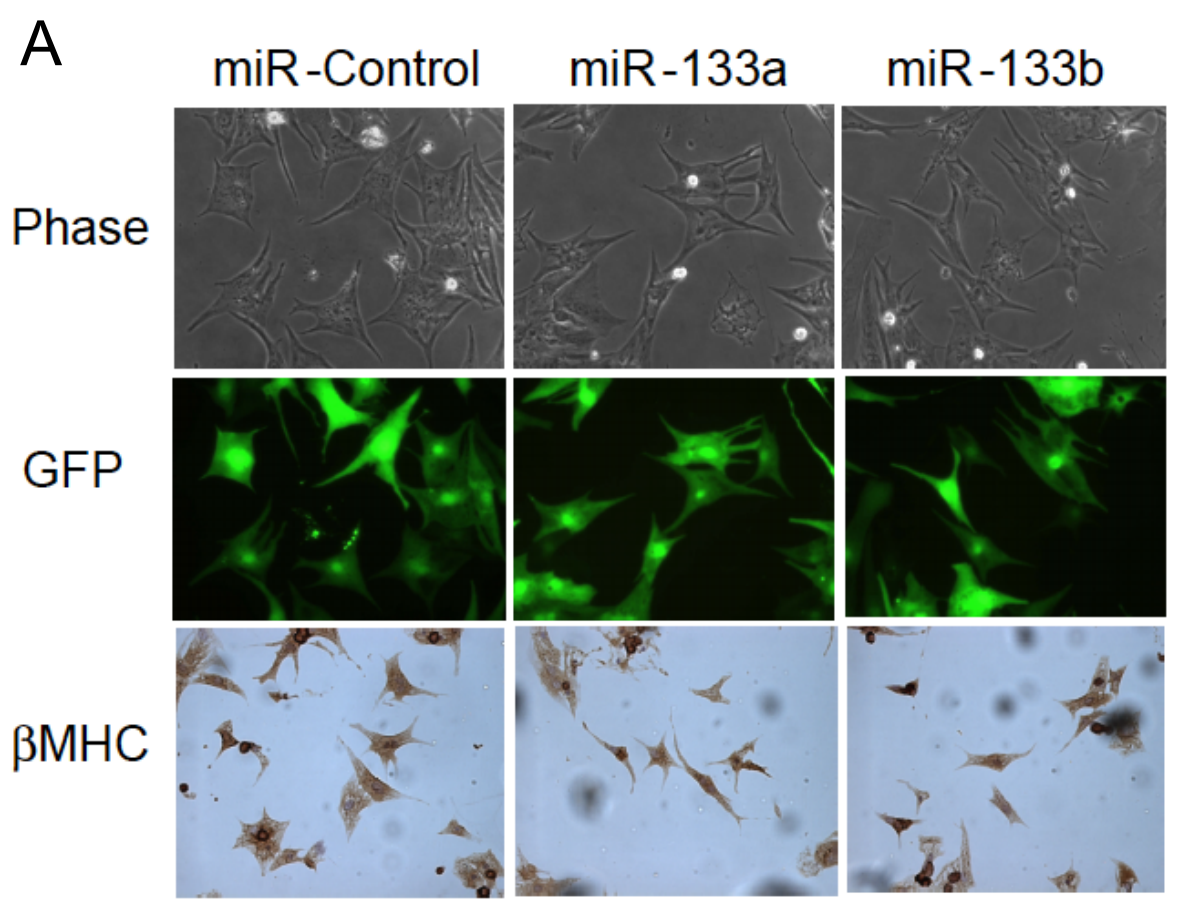

B

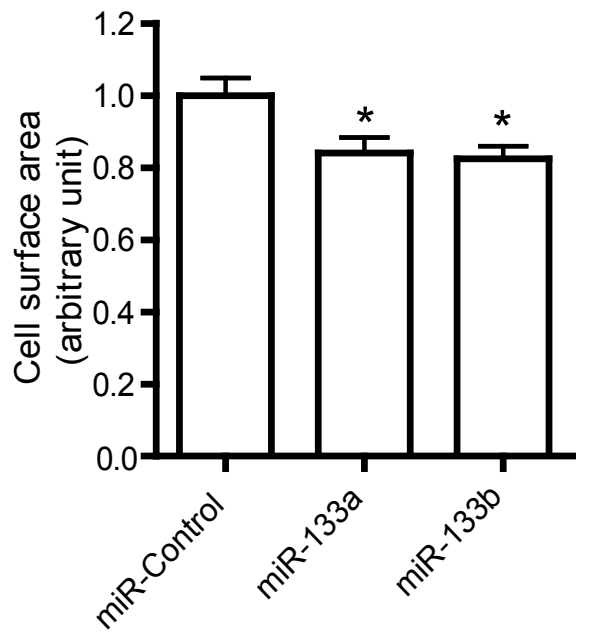

C

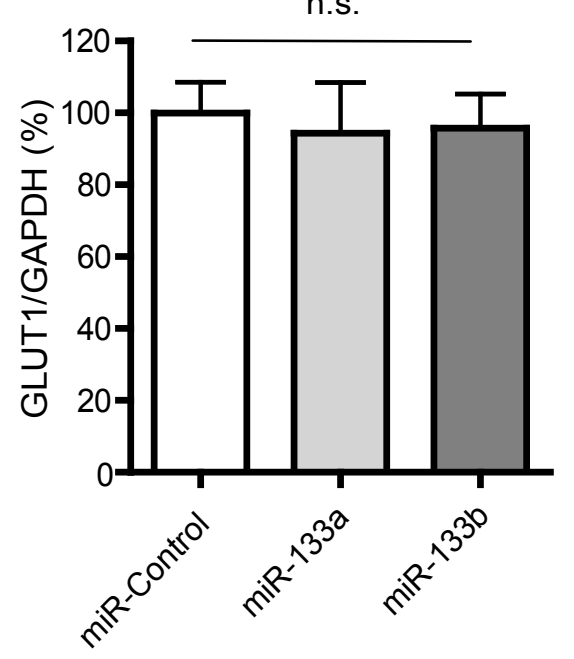

Supplement Fig.1 
A

KLF15 3'UTR

binding site 1

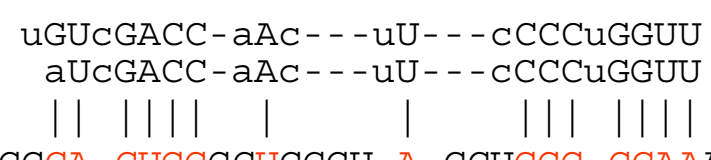

5' $\quad \operatorname{miR}-133 a$

5' $\operatorname{miR}-133 \mathrm{~b}$

Mmu 5' UCUUCCCA-CUGGGCUCCCU-A-CCUGGG- CCAAAGUCAACA

Rno 5' UCCUCCCA-UUGGGCUCCCU-A-CCAGGG-CCAAAGCCAACA

Hsa 5' UUCUCCCA-CUGGGCUCCCCCAUCCUGGG-CCAAGGCCAGAA

binding site 2

UGUCGACCAACUUC-C-CCUGGuu

$5^{\prime}$ aUCGACCAACUUC-C-CCUGGuu 5'

$\operatorname{miR}-133 a$ || ||| ||| | | |||||

$\operatorname{miR}-133 \mathrm{~b}$

Mmu 5' AAGGGCUGCA-CUG- -UGAUGUGAGGACCGCCAUAUGCAAGA

Rno 5' AAGGGCUGUA-CUG--UGGUGCGAGGACCGCCAUAUGCAAGA

Hsa 5' AAGGGCUGCA-CUG--UGGUGUGAGGGUGGCUUUGUCUAAGA

B
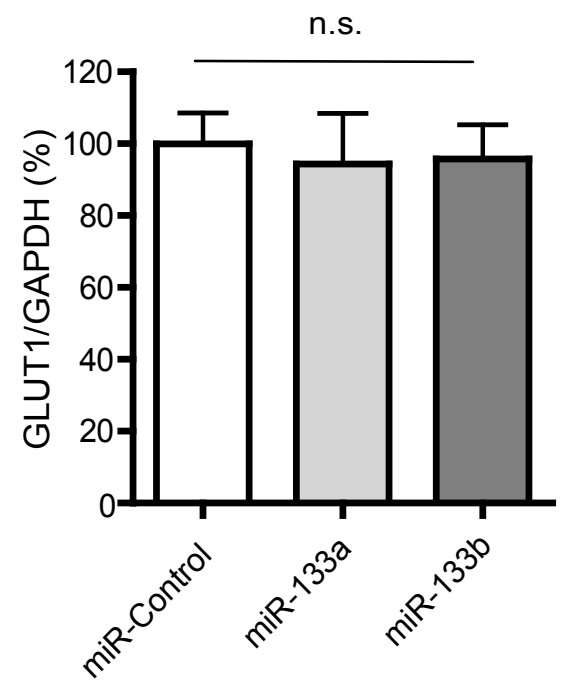
A

Control-decoy (no binding site)

Decoy-miR-133a

(anti-miR-133ax3)

\begin{tabular}{l}
- CMV \\
- CMV \\
\hline
\end{tabular}

Anti-miR-133 Anti-miR-133 Anti-miR-133 AAAAA
B

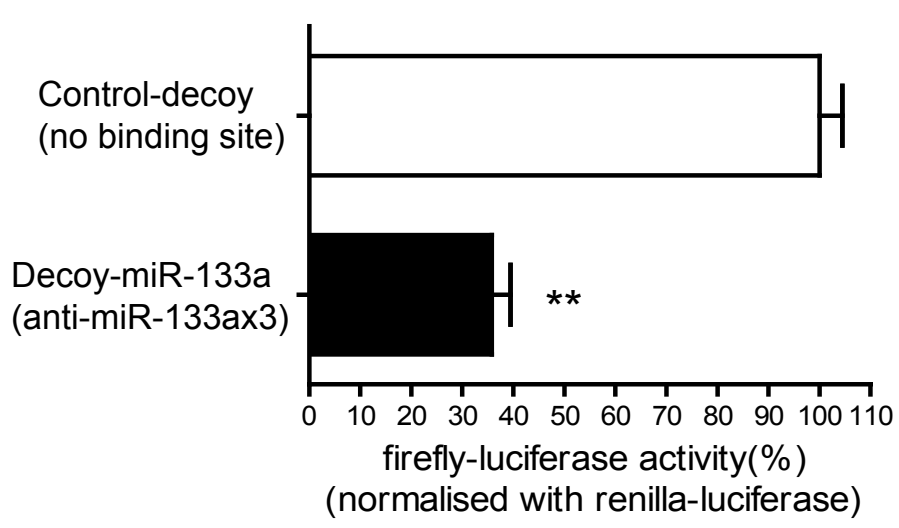

D

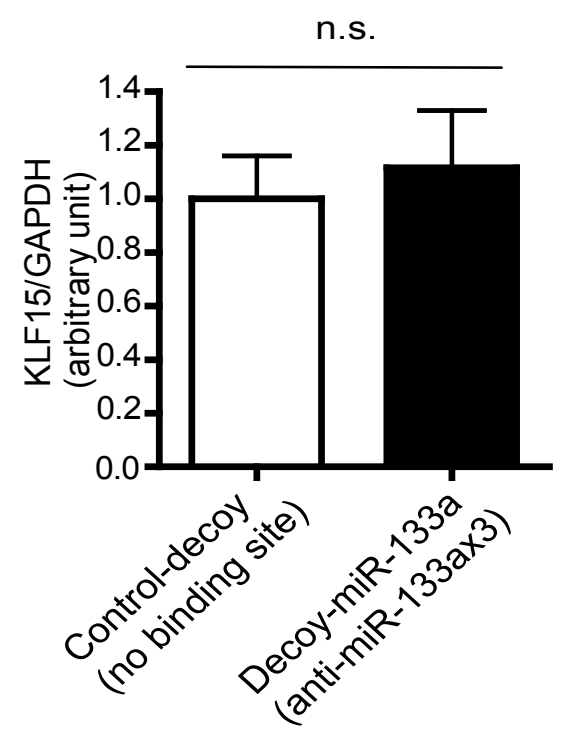

C

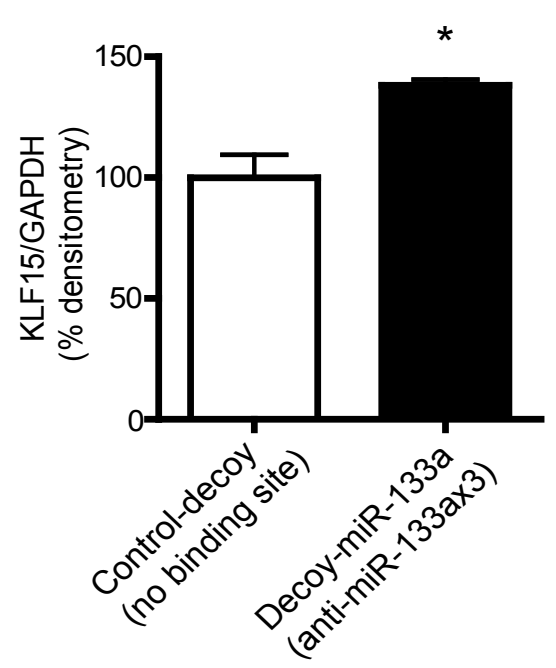

$E$

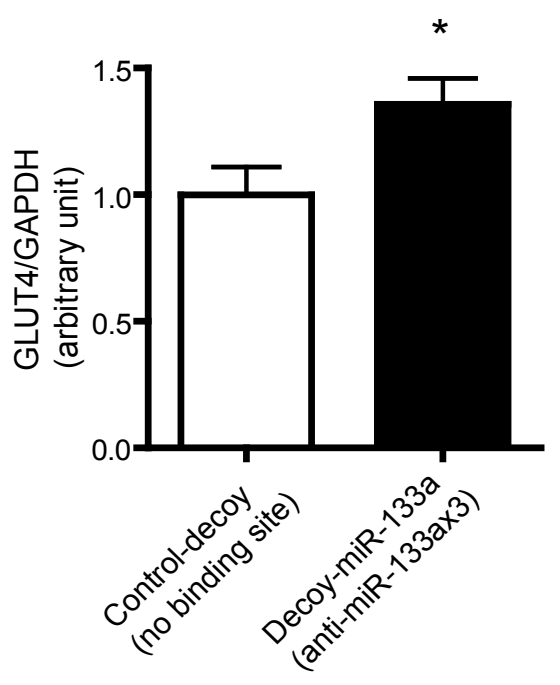

Supplement Fig.3 